\title{
Development of a Method for Identification and Quantification of Sulfadiazine and Pyrimethamine in Serum of Congenital Toxoplasmosis Pediatric Patients
}

\author{
Maria Elena Marson 1,2,3*, Fleitas Ulises 1,2,3, Pérez Montilla Carlos ${ }^{4}$, Prospitti Anabela1,2, \\ Altcheh Jaime 3,4 , Moroni Samanta ${ }^{4}$, Moscatelli Guillermo ${ }^{3,4}$, García Bournissen Facundo5, \\ Mastrantonio Guido ${ }^{1,2,3}$
}

\footnotetext{
${ }^{1}$ Área de Toxicología, Departamento de Ciencias Biológicas, Facultad de Ciencias Exactas, Universidad Nacional de La Plata, La Plata, Provincia de Buenos Aires, Argentina

${ }^{2}$ Laboratorio UPL, Universidad Nacional de La Plata/Comisión de Investigaciones Científicas de la Provincia de Buenos Aires, La Plata, Argentina

${ }^{3}$ Consejo Nacional de Investigaciones Científicas y Técnicas (CONICET), La Plata, Argentina

${ }_{4}^{4}$ Instituto Multidisciplinario de Investigaciones en Patologías Pediátricas (IMIPP-CONICET), Servicio de Parasitología y Chagas, Hospital de Niños “Dr. Ricardo Gutiérrez”, Buenos Aires, Argentina

${ }^{5}$ Division of Paediatric Clinical Pharmacology, Department of Paediatrics, Schulich School of Medicine and Dentistry, Western Ontario University, London, Ontario, Canada

Email: *emarson@biol.unlp.edu.ar
}

\begin{abstract}
How to cite this paper: Marson, M.E., Ulises, F., Carlos, P.M., Anabela, P., Jaime, A., Samanta, M., Guillermo, M., Facundo, G.B. and Guido, M. (2021) Development of a Method for Identification and Quantification of Sulfadiazine and Pyrimethamine in Serum of Congenital Toxoplasmosis Pediatric Patients. $P h$ armacology \& Pharmacy, 12, 155-166. https://doi.org/10.4236/pp.2021.128014
\end{abstract}

Received: April 6, 2021

Accepted: August 24, 2021

Published: August 27, 2021

Copyright $\odot 2021$ by author(s) and Scientific Research Publishing Inc. This work is licensed under the Creative Commons Attribution International License (CC BY 4.0).

http://creativecommons.org/licenses/by/4.0/ (c) (i) Open Access

\begin{abstract}
Infection with Toxoplasma gondii, is one of the most widespread zoonoses in the world. Congenital Toxoplasmosis (CT) is particularly risky due to its fetal complications. Sulfadiazine (SDZ) and Pyrimethamine (PYR) are usually used for CT treatment in Argentina, to prevent morbidity. Due to the lack of commercial pediatric formulations, these must be prepared in the hospital pharmacy. This is the first report of serum concentrations measures in pediatric CT therapy for this combination of drugs. A bioanalytical method was developed for identification and simultaneous quantification of SDZ and PYR by High Performance Liquid Chromatography (HPLC) with UV detection. The validated method was applied to residual serum samples obtained from 6 pediatric patients undergoing treatment with SDZ 42.20 a $93.70 \mathrm{mg} / \mathrm{kg} /$ day and PYR 0.77 a $2.70 \mathrm{mg} / \mathrm{kg} /$ day. Sample pretreatment consisted on a deproteinization step followed by centrifugation and then injection of supernatant. Limit of Detection (LOD) and Quantification (LOQ) were $(0.17 \pm 0.02$ and $0.13 \pm$ $0.02) \mu \mathrm{g} / \mathrm{mL}$ and $(0.46 \pm 0.01$ and $0.36 \pm 0.01) \mu \mathrm{g} / \mathrm{mL}$ for SDZ and PYR respectively, with an appropriate linear range. Concentrations range found were
\end{abstract}


$(<\mathrm{LOD}-162.04 \pm 0.02) \mu \mathrm{g} / \mathrm{mL}$ for SDZ and $(<\mathrm{LOD}-7.30 \pm 0.03) \mu \mathrm{g} / \mathrm{mL}$ for PYR. We developed and validated in real pediatric samples, an acute, precise and low-cost method for quantification of SDZ and PYR using a non-sophisticate chromatographic equipment, suitable for hospital therapeutic monitoring for public health system.

\section{Keywords}

Toxoplasmosis, Bioanalytics, Pediatric Pharmacology, Therapeutic Drug Monitoring, Neglected Diseases

\section{Introduction}

Infection with Toxoplasma gondii, is one of the most widespread zoonoses in the world. The worldwide infection prevalence is estimated at $30 \%$. The infection is acquired mainly by food contaminated with parasite cysts, and is usually asymptomatic. The congenital infection is particularly risky, with rates of mother-to-baby transmission approaching $90 \%$ in the last month of pregnancy. There are few studies on the incidence of Congenital Toxoplasmosis (CT) in Latin America, but the seroprevalence in women of childbearing age is high [1]. Mostly the infection is asymptomatic, and the diagnosis is made by serological screening. Treatment during pregnancy decreased fetal morbidity and sequelae in the child [2]. An early diagnosis followed by treatment of CT in infants provides a better resolution of clinical signs compared to those not treated [3] [4]. Between 10\% and $30 \%$ of prenatal infections result in abortion, death of the newborn or severe clinical signs at birth [5] [6]. However, about $67 \%$ of congenital infections are clinically asymptomatic at birth and may develop symptoms later, predominantly ocular lesions [3] [6].

The current therapy in pediatric patients is protocolized, but due to the absence of pediatric formulations of the drugs, these are prepared in the hospital pharmacy in the form of syrup and at the moment, pharmacological parameters of these drugs have not been corroborated on serum samples for this combination of SDZ and PYR.

Drugs available for the treatment of toxoplasmosis only inhibit the growth of the parasite when it is in the active phase of its life cycle (tachyzoite), not being useful against the cystic or latent form of the parasite (bradyzoites). Most health centers do not hesitate to recommend treatment to infants with confirmed CT. However, to date, there is no controlled study in our country that determines its efficacy, the appropriate therapeutic dose and the optimal duration [6].

Indeed, there are some coincidences about the drugs to be used, but the duration of treatment has been more discussed. Prolonged treatments are associated with a lower rate of sequelae while short treatments have the advantage of reducing drug toxicity. The treatment scheme in Argentina is SDZ $50-100 \mathrm{mg} / \mathrm{kg} / \mathrm{d}$ associated with PYR $1 \mathrm{mg} / \mathrm{kg} / \mathrm{d}$ and folinic acid $5 \mathrm{mg} / 48$ hours. The duration is 
from diagnosis to one year of age with a minimum time of 6 months if the child is older.

PYR (5-(4-Chlorophenyl)-6-ethyl-2,4-diaminopyrimidine) interferes with the synthesis of folic acid by inhibiting dihydropteroate synthase and dihydrofolate reductase and due to poorly studied pharmacological factors, treatment may not be successful.

SDZ (N-amino-N-pyrimidin-2-yl-benzenesulfonamide) is the most active sulfamide against $T$. gondii. It has synergistic activity with PYR but being analogous to the PABA, necessary for the production of parasitic nucleic acids. It is excreted by the kidney, requiring dose adjustment in patients with renal impairment. It is not indicated in patients with glucose deficiency 6-phosphate dehydrogenase (G6PDH) and replaced with clindamycin.

There are few studies in the pediatric population in other countries, where PYR or SDZ on serum samples are measured but with different therapeutic doses or combination of drugs used [7] [8] [9] [10]. A publication describing pharmacokinetic parameters in the pediatric population treated several months for CT with PYR and sulfadoxine, proposes the existence of a wide interindividual variability and at a dose adjusted to weight, plasma concentrations would be unpredictable. Therefore, it has not been possible to establish what plasma concentration or drug combination is the most effective in pediatric CT. Also, the relationship between therapeutic serum concentrations and toxicity is unknown and there are also no studies of interaction with new anticonvulsants or corticosteroids. On the other hand, transfer information of these drugs through the placenta or breast milk is scarce.

All these vacancies can become important working hypotheses. Despite addressing drugs with a long time of use in therapeutics, there is no sufficient pediatric information on the literature. To advance in any of the hypotheses, a simple, fast, precise and clinically adjusted method, such as the one presented in this work, is of great importance as a tool for systematization and improvement of the current pharmacological treatment protocols for this disease.

\section{Methods}

A protocol designed to evaluate the response of a bioanalytical method for identification and simultaneous quantification of SDZ and PYR by High Performance Liquid Chromatography (HPLC) with UV detection was followed. The aim is to validate the HPLC-UV method in order to transfer these capabilities to health institutions that perform therapeutic monitoring of these drugs. Instrumental techniques using HPLC-UV require equipment of medium complexity suitable for the monitoring of pharmacotherapy, available in hospitals and institutions of the public health system in Argentina.

\section{SDZ and PYR Stability over Time}

Accurate standard solutions of SDZ, PYR and a mix of both of them were eva- 
luated at different concentration levels. Duplicate samples of these standard solutions were stored in batches in refrigerator and freezer, respectively. After a defined period of time (between 24 hours and 30 days of storage) each solution was quantified. For every sample, its percentage coefficient of variation (CV\%) intra-day (repeatability) and inter-day (reproducibility) was determined after its storage in refrigerator or freezer. Here, there was no pretreatment step needed for these samples, so as to evaluate only the chromatographic system response and the stability of these standard solutions.

An analogous storage treatment was performed for spiked serum samples at accurate and known concentrations of SDZ, PYR and a mix of both. Stability concentration after storage in the freezer between 24 hours and 30 days was also evaluated for these samples. Here, a pretreatment was needed to extract SDZ and PYR from the serum matrix. Percentage of Recovery (R\%) was calculated for all stored samples and for a fresh spiked serum sample with an accurate concentration as one of those stored, in every day of analysis.

Finally, another stability evaluation was performed in 4 serum samples after 3 cycles of defreeze/freeze at an accurate and known concentrations of SDZ, PYR and a mix of both, followed by extraction and quantification, according to protocols proposed by the National Administration of Medicines, Food and Medical Technology (ANMAT) for the stability of a bioanalytical method [11]. It was proposed as a stablity criterion those samples that presented a CV\% $\leq 15.0 \%$ in all instances.

\section{Materials and Reagents}

Trichloroacetic Acid (TCA) pro analysis grade was purchased from Biopack (Buenos Aires, Argentina). Dimethyl Sulfoxide (DMSO) pro analysis grade was obtained from Anedra (Buenos Aires, Argentina). Chromatographic grade demineralized water $(<0.2 \mu$ siemens) was obtained in our laboratory with ionic interchange resins. Acetonitrile (AcN) and Methanol (Me) (J.T. Baker, USA) HPLCgrade were used. Sulfadiazine (Stanton L_1205050015269/0088) was obtained from the hospital pharmacy and pyrimethamine was obtained from sigma Aldrich.

A Table Top centrifuge (MRC, Scientific Instruments, Argentina) and a rotary evaporator (Heidolph Laborota 4010) equipped with a ROTAVAP valve control equipment were used for the pretreatment procedures. A certified $0.1 \mathrm{mg}$ analytical (Ohaus-Pionner, USA) was used in weighing operations. All micropipettes were calibrated before use. All HPLC solvents were degassed with a vacuum pump (Pascal, Buenos Aires, Argentina). An ultrasonic homogenizer (FAETA, Argentina) was also used on extraction procedures.

\subsection{HPLC Instrumentation and Calculation}

The instrumental analytical procedure for samples measures were performed with an LC system consisting of an HPLC Merck-Hitachi LC-6200A and MerckHitachi UV/Vis L-4250 detector (Japan). Separations were carried out at room 
temperature using a C18 column $5 \mu \mathrm{m}, 100 \mathrm{~mm} \times 4.6 \mathrm{~mm}$ I.D. Lichrospher-100 RP18 (Merck, USA). Samples were injected with a manual injector system with a $20 \mu \mathrm{L}$ sample loop. Peak areas were integrated automatically by Merck-Hitachi D-2500 Chromato-Integrator. All the calculations concerning the quantitative analysis were performed with an external standardization by the measurement of peak areas of a sample specimen series.

Limits of Detection (LOD) were established at 3.3 times of intercept coefficient standard error/slope coefficient ratio. Limits of Quantization (LOQ) were established at nine times of intercept coefficient standard error/slope coefficient ratio. Accuracy and precision of the assays were calculated based on the analysis of three replicates for each level of the standard curve. Total uncertainty was calculated as the sum of accuracy and precision.

\subsection{Standard Solutions}

Standard solutions of SDZ and PYR were prepared separately. For SDZ standard solution, $0.0507 \mathrm{~g}$ were dissolved in $10 \mathrm{~mL}$ of DMSO. To complete dissolution it was accurately diluted to $25.00 \mathrm{~mL}$ in a calibrated volumetric flask with a solvent mix of Me:water (50:50) to obtain a $2028.0 \mu \mathrm{g} / \mathrm{mL}$ SDZ solution. For PYR standard solution, $0.0297 \mathrm{~g}$ were dissolved in $10 \mathrm{~mL}$ of $\mathrm{AcN}$ and then accurately diluted to $25.00 \mathrm{~mL}$ in a calibrated volumetric flask with the same solvent mix used for SDZ to obtain an $1189.0 \mu \mathrm{g} / \mathrm{mL}$ PYR solution. These standard solutions and dilutions of them were used for analyze SDZ and PYR stability over time (Table 1).

Also, variable volumes of these standard solutions were added to drug-free serum to obtain matrix standards of $1000 \mu \mathrm{L}$ volume for calibration curves. These

Table 1. Drug standard solutions evaluated in aqueous and serum matrix.

\begin{tabular}{cccccc}
\hline Standard & SDZ $(\mu \mathrm{g} / \mathrm{mL})$ & PYR $(\mu \mathrm{g} / \mathrm{mL})$ & Standard & SDZ $(\mu \mathrm{g} / \mathrm{mL})$ & PYR $(\mu \mathrm{g} / \mathrm{mL})$ \\
\hline SDZ St 1 & 244.00 & - & HC SDZ & 154.57 & - \\
SDZ St dil 1 & 24.40 & - & HC SDZ + PYR & 154.57 & 8.08 \\
SDZ St 2 & 240.00 & - & LC SDZ & 9.76 & - \\
SDZ St dil 2 & 24.00 & - & LC SDZ + PYR & 9.76 & 8.08 \\
SDZ St 3 & 1189.0 & - & HC PYR & 80.80 & - \\
SDZ St dil 3 & 118.90 & - & HC PYR + SDZ & 80.80 & 24.00 \\
PYR St 1 & - & 404.00 & LC PYR & 10.10 & - \\
PYR St dil 1 & - & 40.40 & LC PYR + SDZ & 10.10 & 24.00 \\
\hline
\end{tabular}

SDZ St: Sulfadiazine Standard Solution; SDZ St dil: dilution 1/10 of Sulfadiazine Standard Solution; PYR St: Pyrimethamine Standard Solution; PYR St dil: dilution 1/10 of Pyrimethamine Standard Solution; HC SDZ: High Sulfadiazine Concentration on Serum; HC SDZ + PYR: High Sulfadiazine Concentration with Pyrimethamine on Serum; LC SDZ: Low Sulfadiazine Concentration on Serum; LC SDZ + PYR: Low Sulfadiazine Concentration with Pyrimethamine on Serum. HC PYR: High Pyrimethamine Concentration on Serum; HC PYR + SDZ: High Pyrimethamine Concentration with Sulfadiazine on Serum; LC PYR: Low Pyrimethamine Concentration on Serum; LC PYR + SDZ: Low Pyrimethamine Concentration with Sulfadiazine on Serum. 
curves were made in triplicate for the lowest concentration point and duplicated for the rest of the points. Duplicated drug-free serum samples as a control specimen were included on calibration curves. Concentration points for both drugs analysed were $(0.05 ; 0.56 ; 5.60 ; 70.50 ; 170.10 ; 240.66) \mu \mathrm{g} / \mathrm{mL}$ for SDZ and $(0.03$; $0.51 ; 0.91 ; 2.42 ; 7.07 ; 16.10) \mu \mathrm{g} / \mathrm{mL}$ for PYR (Table 2 ). In all cases the volume of the standard solution added to the serum matrix did not exceed $20 \%$ off its volume to minimize dilution effects. For control specimen a $20 \%$ serum sample volume of the solvent used for standard solutions was added.

\subsection{Serum Samples}

Residual serum samples were obtained from six pediatric patients treated for TC from a clinical study with PYR $(0.77$ a $2.70 \mathrm{mg} / \mathrm{kg} /$ day $)$, aged between 33 days and 3 year old. Samples were stored at $-20^{\circ} \mathrm{C}$ until analysis.

The clinical study and its informed consent for the use of the samples were approved by the institutional ethics committee of the Ricardo Gutiérrez Children's Hospital (RGCH).

\subsection{Sample Pretreatment and Chromatographic Conditions}

All $1000 \mu \mathrm{L}$ samples were deproteinized with $50 \mu \mathrm{L}$ of TCA (30\% p/v), vortexed for one minute, and sonicated for five minutes. The mixture was then centrifuged at $8000 \mathrm{~g}$ for another five $5 \mathrm{~min}$. After this, $300 \mu \mathrm{L}$ of the supernatant were separated on an eppendorf before injecting it into the HPLC system.

The HPLC analysis was performed by a gradient elution in a Reverse Phase (RP) mode. The mobile phase composition varied from $90 \%$ to $50 \%$ of water ( $1 \%$ $\mathrm{v} / \mathrm{v}$ of formic acid) with methanol from $5 \%$ to $45 \%$ and $5 \%$ of acetonitrile that remained constant throughout the run. The flow ranged was 0.8 to $1.0 \mathrm{~mL} / \mathrm{min}$ and the total running time was fourteen minutes. All solvents were filtered through a $0.45 \mu \mathrm{m}$ nylon membrane and degassed before use. The maximum UV absorption found for simultaneous identification of SDZ and PYR was at $273 \mathrm{~nm}$, so this wavelength was chosen for the method. A value of 0.030 absorbance units (a.u.) threshold was used. Duplicate injections were made for all samples to test

Table 2. Concentration points used for calibration curves.

\begin{tabular}{ccc}
\hline Mta & SDZ $(\mu \mathrm{g} / \mathrm{mL})$ & PYR $(\mu \mathrm{g} / \mathrm{mL})$ \\
\hline CS a/b & - & - \\
St $1 \mathrm{a} / \mathrm{b} / \mathrm{c}$ & 0.05 & 0.03 \\
St $2 \mathrm{a} / \mathrm{b}$ & 0.56 & 0.51 \\
St $3 \mathrm{a} / \mathrm{b}$ & 5.60 & 0.91 \\
St 4 a/b & 70.50 & 2.42 \\
St $5 \mathrm{a} / \mathrm{b}$ & 170.10 & 7.07 \\
St 6 a/b & 240.66 & 16.10 \\
\hline
\end{tabular}

CS: Control Specimen; St 1 to 6: standard points at six concentration levels; a/b/c: indicates triplicate samples; a/b: indicates duplicate samples. 
reproducibility of the detector response at each concentration level. Peak area was plotted against concentration to obtain calibration graphs. Linear regression and a one way analysis of variance were applied to calculate calibration equation and statistical correlation coefficients.

\section{Results}

Table 3 and Figure 1 show intraday CV\% (repeatability), between duplicates of standard solutions in refrigerator and freezer after each storage time. The last two columns of the table present the CV\% inter-day (reproducibility) for each standard in solvent or in serum for storage in refrigerator or freezer respectively. Higher concentrated standard solutions presented lower CV\% than those diluted. Although the reproducibility in no case exceeds the maximum CV\% proposed as acceptable, it was found that the standard solutions stored in the refrigerator within the period evaluated, had lower CV\% than those stored in the freezer. This difference may be due to factors such as the decrease in the solubility of drugs at low temperatures associated with some systematic error in the homogenization prior to injection into the chromatographic equipment.

No evaluation on serum was made for sample storage in the refrigerator because it is well known that serum samples refrigerator storage is not recommended for periods longer than 24 hours. For this reason, matrix standard solutions storage behavior were only studied in freezer. Here, there were no significant

Table 3. Sulfadiazine and pyrimethamine stability over time.

\begin{tabular}{cccccccc}
\hline Sample & $\begin{array}{c}\text { Day 1 } \\
\text { CV\% }\end{array}$ & $\begin{array}{c}\text { Day 7 } \\
\text { CV\% }\end{array}$ & $\begin{array}{c}\text { Day 14 } \\
\text { CV\% }\end{array}$ & $\begin{array}{c}\text { Day 23 } \\
\text { CV\% }\end{array}$ & $\begin{array}{c}\text { Day 27 } \\
\text { CV\% }\end{array}$ & $\begin{array}{c}\text { CV\% } \\
\text { (Fridge) }\end{array}$ & $\begin{array}{c}\text { CV\% } \\
\text { (Freezer) }\end{array}$ \\
\hline SDZ St 1 & 3.31 & 3.80 & 6.44 & 6.20 & 6.50 & 3.08 & 10.9 \\
SDZ St 2 & 2.11 & 6.74 & 4.29 & 2.31 & 4.33 & 4.75 & 8.12 \\
SDZ Dil St 1 & 10.60 & 3.80 & 2.67 & 4.42 & 5.78 & 9.02 & 7.90 \\
SDZ Dil St 2 & 7.30 & 8.84 & 2.12 & 5.10 & 4.09 & 10.30 & 10.70 \\
PYR St 1 & 10.82 & 10.01 & 9.73 & 10.32 & 9.78 & 10.73 & 10.69 \\
PYR Dil St 1 & 11.32 & 10.80 & 11.16 & 10.68 & 10.04 & 10.90 & 11.25 \\
LC SDZ & 6.90 & 13.70 & 3.40 & 3.40 & 0.60 & - & 10.50 \\
LC SDZ + PYR & 7.80 & 12.90 & 3.20 & 10.1 & 5.90 & - & 9.70 \\
HC SDZ & 1.30 & 0.50 & 1.50 & 7.50 & 5.90 & - & 11.46 \\
HC SDZ + PYR & 2.10 & 9.80 & 1.70 & 3.90 & 4.80 & - & 10.68 \\
HC PYR & 10.75 & 11.20 & 11.20 & 9.70 & 10.90 & - & 11.40 \\
HC PYR + SDZ & 9.26 & 8.90 & 12.30 & 11.65 & 11.40 & - & 13.75 \\
LC PYR & 7.98 & 13.32 & 13.70 & 12.90 & 11.00 & - & 10.92 \\
LC PYR + SDZ & 7.45 & 7.56 & 9.89 & 10.20 & 10.10 & - & 11.62 \\
\hline
\end{tabular}

Columns 2 to 5 shows intra-day (repeatability) percentage Coefficient of Variation (CV\%) between duplicates in refrigerator and freezer after each storage time. The last two columns show inter-day (reproducibility) CV\% for storage in refrigerator or freezer respectively. Sample References were presented on Table 1. 


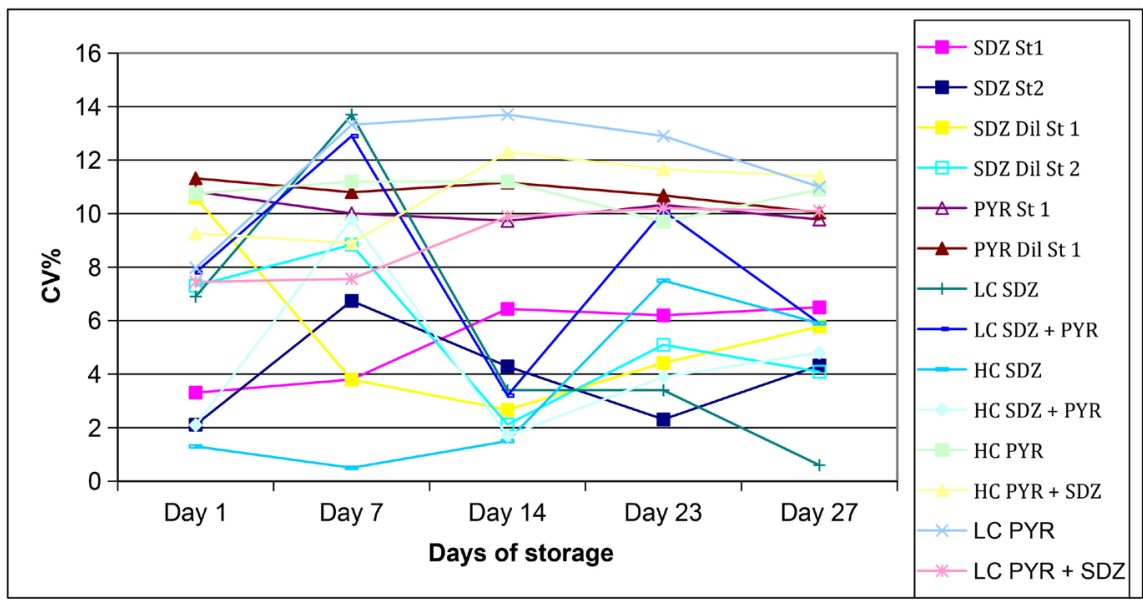

Figure 1. Intra-day CV\% (repeatability) between duplicates of standard solutions in refrigerator and freezer after each storage time.

differences in CV\% or R\% between high or low concentrations of both SDZ and PYR. Also, the presence of the two drugs together in the matrix did not cause analytical interference or significant increases in its CV\% or decreases on R\%.

The CV\% obtained for serum samples after 3 cycles of defrosting/freezing are presented in Table 4. There, it can be observed that the CV\% are slightly higher for PYR than for SDZ (average CV\% of 12.88 for the SDZ and 14.03 for the PYR) but they do not exceed the values proposed as acceptable in any case.

Calibration curves for simultaneous detection and quantification of SDZ and PYR were made on three different days with six concentration levels. Total retention time range for gradient mode used on chromatographic analysis was (5.01 - 5.36) minutes for SDZ and (12.16 - 12.60) minutes for PYR. Also, SDZ and PYR retention time ratio had a range of $0.41-0.43$ and a total run time was 14 minutes per sample. The LOD and LOQ were $(0.17 \pm 0.02$ and $0.13 \pm 0.02)$ $\mu \mathrm{g} / \mathrm{mL}$ and $(0.46 \pm 0.01$ and $0.36 \pm 0.01) \mu \mathrm{g} / \mathrm{mL}$ for SDZ and PYR respectively. The validated method had a linear range of $(<\mathrm{LOQ}-210.00 \pm 0.02) \mu \mathrm{g} / \mathrm{mL}$ for SDZ and $(<\mathrm{LOQ}-15.05 \pm 0.02) \mu \mathrm{g} / \mathrm{mL}$ for PYR. A summary of calibration curve statistical parameters are presented on Table 5.

Chromatographic parameters such as resolution, selectivity, and peak asymmetry were satisfactory for SDZ and PYR determination with this method. In addition, analytes showed no decomposition products detectable in chromatograms profiles. Figure 2 presents a characteristic chromatogram profile for the proposed RP gradient chromatographic system for the detection and quantification of SDZ and PYR in (a) standard solutions mix (b) serum extracts for SDZ and PYR.

\section{SDZ and PYR on Pediatric Samples}

With the validated method, SDZ and PYR were measured in a set of 30 samples from 6 pediatric patients who participated in a clinical study to evaluate the pharmacokinetics of both drugs on serum samples. The concentrations range 
Table 4. The CV\% obtained for serum samples at different concentrations after 3 cycles of defrosting/frizzing are presented here.

\begin{tabular}{ccccc}
\hline Sample & CV\% for SDZ & CV\% for PYR & SDZ $(\mu \mathrm{g} / \mathrm{mL})$ & PYR $(\mu \mathrm{g} / \mathrm{mL})$ \\
\hline Serum A & 14.0 & 13.78 & 118.9 & 24.0 \\
Serum B & 13.30 & 12.84 & 10.1 & 24.0 \\
Serum C & 11.98 & 14.59 & 118.9 & 24.0 \\
Serum D & 12.24 & 14.90 & 10.1 & 24.0 \\
\hline
\end{tabular}

Table 5. Statistical parameters found for simultaneous calibration curve for SDZ and PYR.

\begin{tabular}{ccccc}
\hline & SDZ & PYR & R $^{2}$ & Linear range \\
\hline LOD $\mu \mathrm{g} / \mathrm{mL}$ & $(0.13 \pm 0.02)$ & $(0.17 \pm 0.02)$ & 0.97797 & $(0.36-15.05)$ \\
$\mathrm{LOQ} \mu \mathrm{g} / \mathrm{mL}$ & $(0.36 \pm 0.01)$ & $(0.46 \pm 0.01)$ & 0.97566 & $(0.46-210.00)$ \\
$\mathrm{R} \%$ & $89 \pm 2$ & $86 \pm 4$ & - & - \\
$\mathrm{CV} \%$ interday & 3.1 & 4.4 & - & - \\
Accuracy \% & $97 \pm 2$ & $94 \pm 2$ & - & - \\
\hline
\end{tabular}

LOD: Limit of detection; LOQ: Limit of quantification; R (\%): Percentage of recovery; CV\% interday: inter-day (reproducibility) percentage coefficient of variation; $\mathrm{R}^{2}$ : R-squared value.

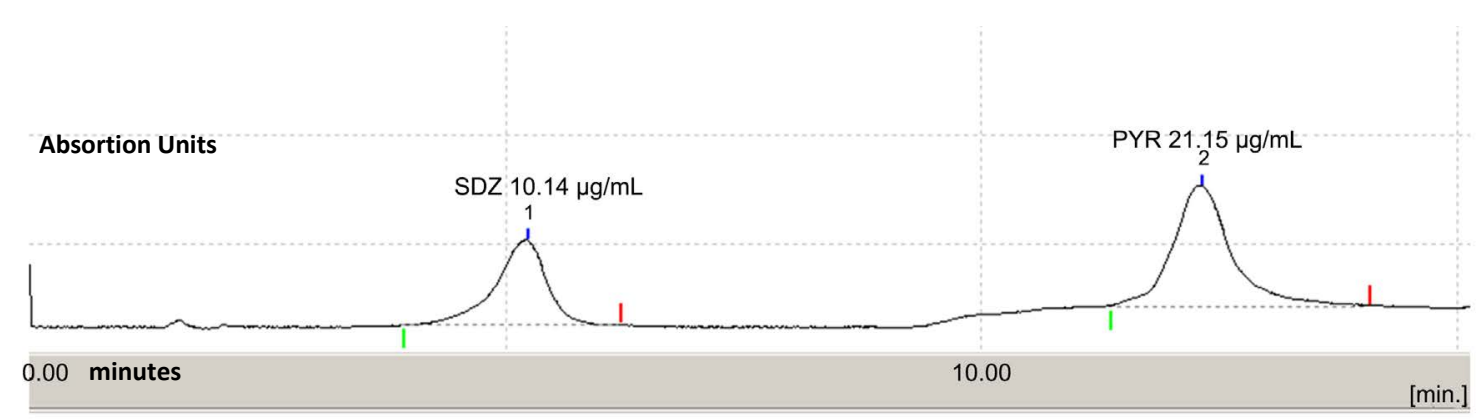

(a)

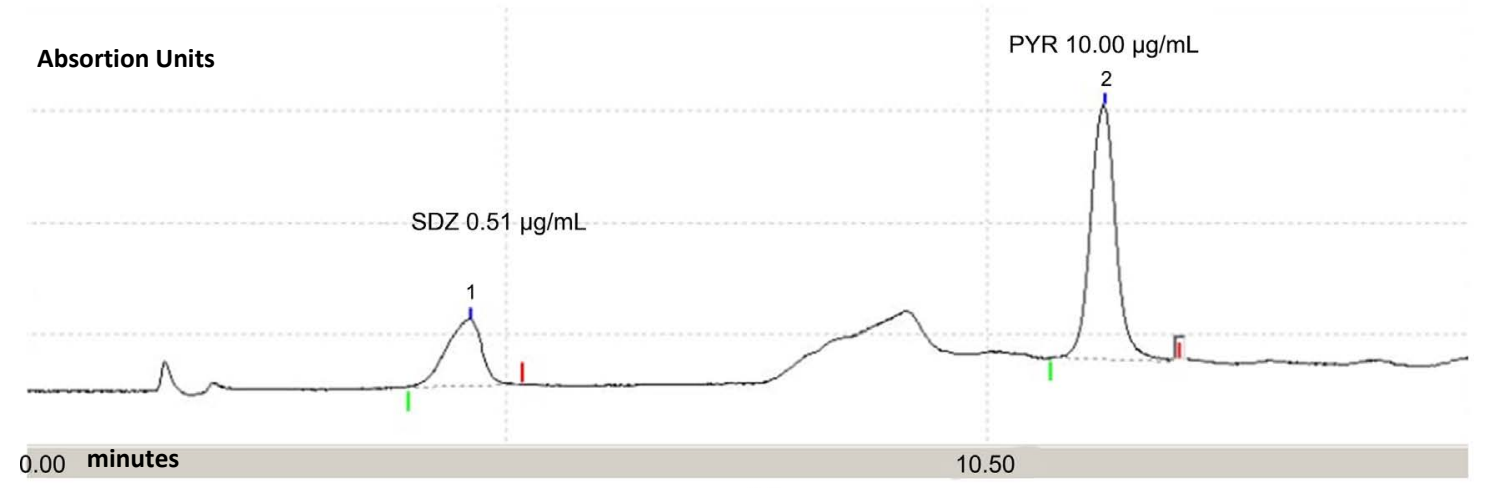

(b)

Figure 2. Characteristic chromatogram for the validated method for SDZ and PYR quantification in (a) standard solution, (b) serum extract.

found were $(<\mathrm{LOD}-162.04 \pm 0.02) \mu \mathrm{g} / \mathrm{mL}$ for SDZ and $(<\mathrm{LOD}-7.30 \pm 0.03)$ $\mu \mathrm{g} / \mathrm{mL}$ for PYR. It should be noted that there is no average value informed for 
each drug measure. The reason is that samples were taken at different times post dose for use in pharmacokinetics so report an average value has no clinical meaning or bioanalytical interest here. In this sense, these measures have the purpose of validating the technique and its application in a real clinical context. The drug stability determinations, plus the parameters evaluated in the validation of the method as a whole, configure measures of the robustness of the method for its use in clinical studies and therapeutic monitoring.

\section{Discussion}

The two drugs analytically evaluated on this study (SDZ and PYR), are currently available for the treatment of TC on public health Institutions in Argentina. However, serum concentrations coming from pediatric formulations prepared in hospital pharmacy have not been quantified before. Until now, there were no validated methods by HPLC/UV developed for the simultaneous detection of these drugs nor doses. In this sense, it was possible to corroborate stability of these drugs on different matrices and its dependence on sample storage period between refrigerator and freezer. This proves the robustness needed for a method suitable for therapeutic monitoring, pharmacokinetics or toxicokinetics.

In literature, there is a developed method for determination of PYR, sulfadoxine, mefloquine, and ibuprofen by HPLC/UV for determination of these drugs in raw materials and dosage of pharmaceutical formulations but SDZ was not included [12]. Also, there are methods developed for SDZ and its hydroxy metabolite and its quantification by reverse phase HPLC [13], and there are others for PYR by HPLC and fluorescence detection, but applied to the malaria pharmacotherapy [14] or for TC but with sulfadoxine instead of SDZ also by HPLC/UV.

There is an interest in the determination of clinically significant serum range of SDZ and PYR concentrations. To our knowledge, there are two different scenarios: adult serum concentration and pediatric serum concentrations. The LOQ of the method described here for both SDZ and PYR, seems to be appropriate in pediatric contexts. Most of the pediatric samples obtained were of $1.00 \mathrm{~mL}$ or less volume of serum. In this sense, minor sample volume may imply a decrease in the sensibility of the method, so there is a compromise between these two variables, also attending that blood samples volumes in pediatrics are normally smaller than in adults. In this sense, it is important to note that pediatric samples analysed for validation procedures were not specially taken. Instead of that, residual volumes of serum were used from an ongoing clinical study.

The development of HPLC methods for determination of drugs has received considerate attention in recent years because of their importance in the quality control of drugs and drug products. The goal of this study was to develop an accurate, precise, and low cost HPLC method for quantification of SDZ and PYR simultaneously, using the most commonly employed C-18 column with UV detection.

Instrumental techniques using HPLC-UV require equipment of medium complexity suitable for the monitoring of pharmacotherapy, available in hospitals 
and institutions of the public health system, giving its advantages over others reported HPLC methods for determination of some of these two drugs but through more expensive and sophisticated detection systems [15].

\section{Conclusions}

We developed and validated in real pediatric samples, an acute, precise and low-cost method for quantification of SDZ and PYR using a non-sophisticate chromatographic equipment, suitable for hospital therapeutic monitoring for public health system.

This is the first report of serum concentrations measures in pediatric CT therapy for this combination of drugs.

\section{Funding and Acknowledgements}

The authors would like to gratefully acknowledge the financial support and the doctoral and postdoctoral scholarships received from Consejo Nacional de Investigaciones Científicas y Técnicas (CONICET), Agencia Nacional de Promoción Científica y Tecnológica (ANPCyT) and Comisión de Investigaciones Científicas de la provincia de Buenos Aires (CICpBA).

\section{Compliance with Ethical Standards}

The clinical study protocol and its informed consent for the use of human samples were approved by the Institutional Ethics Committee of the Ricardo Gutiérrez Children's Hospital.

\section{Conflicts of Interest}

The authors declare no conflicts of interest regarding the publication of this paper.

\section{References}

[1] Durlach, R., Kaufer, F., Carral, L., Freuler, C., Ceriotto, M., Rodríguez, M., et al. (2008) Consenso Argentino de Toxoplasmosis Congénita. Medicina (Buenos Aires), 68, 75-87.

[2] de Infectología, C. (1999) Consenso de infecciones perinatales. Argentine Archive of Pediatrics, 97, 33-37.

[3] McAuley, J., Boyer, K.M., Patel, D., Mets, M., Swisher, C., Roizen, N., Wolters, C., Stein, L., Stein, M., Schey, W., Remington, J., Meier, P., Johnson, D., Heydeman, P., Holfels, E., Withers, S., Mack, D., Brown, C., Patton, D. and McLeod, R. (1994) Early and Longitudinal Evaluations of Treated Infants and Children and Untreated Historical Patients with Congenital Toxoplasmosis: The Chicago Collaborative Treatment Trial. Clinical Infectious Diseases, 18, 38-72.

https://doi.org/10.1093/clinids/18.1.38

[4] Altcheh, J., Diaz, N.S., Pepe, C.M., Martin, V., Nigro, M., Freilij, H. and Angel, S.O. (2006) Kinetic Analysis of the Humoral Immune Response Against 3 Toxoplasma gondii-Recombinant Proteins in Infants with Suspected Congenital Toxoplasmosis. Diagnostic Microbiology and Infectious Disease, 56, 161-165.

https://doi.org/10.1016/j.diagmicrobio.2006.03.012 
[5] Tenter, A., Heckeroth, A.R. and Weiss, L.M. (2000) Toxoplasma gondii. From Animals to Humans. International Journal for Parasitology, 30, 1217-1258. https://doi.org/10.1016/S0020-7519(00)00124-7

[6] Olariu, T., Remington, J.S., McLeod, R., Alam, A. and Montoya, J.G. (2011) Severe Congenital Toxoplasmosis in the United States: Clinical and Serologic Findings in Untreated Infants. The Pediatric Infectious Disease Journal, 30, 1056-1061. https://doi.org/10.1097/INF.0b013e3182343096

[7] Hezard, N., Marx-Chemla, C., Foudrinier, F., Villena, I., Quereux, C., Leroux, B., Dupouy, D., Talmud, M. and Pinon, J.M. (1997) Prenatal Diagnosis of Congenital Toxoplasmosis in 261 Pregnancies. Prenatal Diagnosis, 17, 1047-1054. https://doi.org/10.1002/(SICI)1097-0223(199711)17:11<1047::AID-PD192>3.0.CO;2-Q

[8] Mcleod, R., Mack, D., Foss, R., Boyer, K., Withers, S., Levin, S. and Hubbell, J. (1992) Levels of Pyrimethamine in Sera and Cerebrospinal and Ventricular Fluids from Infants Treated for Congenital Toxoplasmosis. Toxoplasmosis Study Group. Antimicrobial Agents and Chemotherapy, 36, 1040-1048. https://doi.org/10.1128/AAC.36.5.1040

[9] Gilbert, R., Dunn, D., Wallon, M., Hayde, M., Prusa, A., Lebech, M., Kortbeek, T., Peyron, F., Pollak, A. and Petersen, E. (2001) Ecological Comparison of the Risks of Mother-to-Child Transmission and Clinical Manifestations of Congenital Toxoplasmosis according to Prenatal Treatment Protocol. Epidemiology \& Infection, 127, 113-120.

https://doi.org/10.1017/S095026880100560X

[10] Corvaisier, S., Charpiat, B., Mounier, C., Wallon, M., Leboucher, G., Al Kurdi, M., Chaulet, J.-F. and Peyron, F. (2004) Population Pharmacokinetics of Pyrimethamine and Sulfadoxine in Children Treated for Congenital Toxoplasmosis. Antimicrobial Agents and Chemotherapy, 48, 3794-3800.

https://doi.org/10.1128/AAC.48.10.3794-3800.2004

[11] ANMAT Provision No. 2819/04, No. 4844/05 and No. 5040/06.

[12] Arayne, M.S., Sultana, N., Siddiqui, F.A., Naseem, S. and Qureshi, F. (2010) Simultaneous Determination of Pyrimethamine, Sulfadoxine, Mefloquine, and Ibuprofen in Pharmaceutical Formulations by RP-HPLC. Medicinal Chemistry Research, 19, 1043-1054. https://doi.org/10.1007/s00044-009-9250-4

[13] Vree, T. B., van de Ven, E.S., van Wissen, C.P.W.G.M.V., Baars, A.M., Swolfs, A., van Galen, P.M. and Amatdjais-Groenen, H. (1995) Isolation, Identification and Determination of Sulfadiazine and Its Hydroxy Metabolites and Conjugates from Man and Rhesus Monkey by High-Performance Liquid Chromatography. Journal of Chromatography B: Biomedical Sciences and Applications, 670, 111-123.

https://doi.org/10.1016/0378-4347(95)00163-D

[14] Timm, U. (1982) Determination of Pyrimethamine in Human Plasma after Administration of Fansidar or Fansidar-Mefloquine by Means of High-Performance Liquid Chromatography with Fluorescence Detection. Journal of Chromatography B: Biomedical Sciences and Applications, 230, 107-114. https://doi.org/10.1016/S0378-4347(00)81435-8

[15] Johannessen, J.K., Christiansen, I., Schmidt, D.R., Petersen, E. and Hansen, S.H. (2005) Simultaneous Determination of Pyrimethamine, Sulfadiazine and N-Acetyl-Sulfadiazine in Plasma for Monitoring Infants in Treatment of Congenital Toxoplasmosis. Journal of Pharmaceutical and Biomedical Analysis, 36, 1093-1098.

https://doi.org/10.1016/j.jpba.2004.09.001 